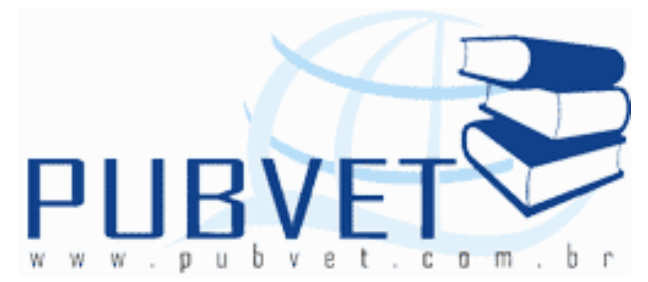

PUBVET, Publicações em Medicina Veterinária e Zootecnia.

\title{
Resposta de Sesbania sesban à inoculação de micorrizas arbusculares
}

Newton de Lucena Costa ${ }^{1}$, Valdinei Tadeu Paulino ${ }^{2}$, Rogério Sebastião Correia da Costa ${ }^{3}$, Ricardo Gomes de Araújo Pereira ${ }^{4}$, João Avelar Magalhães ${ }^{5}$, Claudio Ramalho Townsend ${ }^{4}$, Maria Jucineide Aguiar Rodrigues ${ }^{6}$

${ }^{1}$ Eng. Agr., M.Sc., Embrapa Roraima. Doutorando em Agronomia/Produção Vegetal, UFPR, Curitiba, Paraná.

${ }^{2}$ Eng. Agr., Ph.D, Instituto de Zootecnia, Nova Odessa, SP.

${ }^{3}$ Eng. Agr., D.Sc., Pesquisador da Embrapa Rondônia. Porto Velho, Rondônia.

${ }^{4}$ Zootecnista, D.Sc., Embrapa Rondônia. Porto Velho, Rondônia.

${ }^{5}$ Méd. Vet., D.Sc., Pesquisador da Embrapa Meio-Norte/UEP de Parnaíba. Parnaíba, Piauí.

${ }^{6}$ Graduanda em Biologia da UFPI, Parnaíba, Piauí.

\section{Resumo}

Avaliou-se o efeito da inoculação de micorrizas arbusculares (Glomus mossaea, G. fasciculatum, G. macrocarpum, G. etunicatum, Acaulospora muricata e Scutellospora heterogama), sobre o crescimento e composição química de Sesbania sesban. Entre os fungos avaliados, os mais eficientes na produção de matéria seca foram S. heterogama e A. muricata, os quais proporcionaram incrementos de 155 e 140\%, respectivamente, em relação ao tratamento sem inoculação. As taxas de colonização radicular foram afetadas pelas diferentes espécies de micorrizas arbusculares. Os maiores valores foram registrados com 
COSTA, N.L. et al. Resposta de Sesbania sesban à inoculação de micorrizas arbusculares. PUBVET, Londrina, V. 6, N. 9, Ed. 196, Art. 1314, 2012.

a inoculação de G. fasciculatum, S. heterogama e G. mossaea. Os maiores teores de nitrogênio (N) foram obtidos com a inoculação de G. mossaea e $G$. etunicatum, enquanto que plantas micorrizadas por $S$. heterogama e $A$. muricata apresentaram as maiores quantidades absorvidas de $\mathrm{N}$. Para os teores de fósforo $(P)$, os maiores valores foram verificados com a inoculação de G. mossaea e A. muricata, no entanto a sua maior absorção ocorreu com a inoculação de $S$. heterogama e $A$. muricata. Os resultados obtidos evidenciam que a inoculação de micorrizas arbusculares incrementou significativamente os rendimentos de MS, teores e quantidades absorvidas de $\mathrm{N}$ e $\mathrm{P}$, sendo que os fungos mais efetivos, em termos de rendimento de matéria seca, foram $S$. heterogama e $A$. muricata.

Palavras-chave: colonização radicular, fósforo, matéria seca, nitrogênio

\section{Response of Sesbania sesban to arbuscular mycorrhiza inoculation}

\section{Abstract}

The effects of arbuscular mycorrhiza inoculation - Glomus mossaea, G. fasciculatum, G. macrocarpum, G. etunicatum, Acaulospora muricata and Scutellospora heterogama, on dry matter (DM) yield, and chemical composition of Sesbania sesban were evaluated under greenhouse conditions. The most effectives fungi on DM yields were $S$. heterogama and $A$. muricata, which provided increases of 155 and $140 \%$, in relation to the control. Root colonization was affected by arbuscular mycorrhiza inoculation. Plants inoculated with $G$. fasciculatum, S. heterogama and G. mossaea had the highest levels of root colonization. The highest $\mathrm{N}$ contents were obtained with G. mossaea and G. etunicatum, while plants inoculated with $S$. heterogama and $A$. muricata exhibited higher $\mathrm{N}$ uptake. The highest $\mathrm{P}$ contents were recorded with the inoculation of $G$. mossaea and $A$. muricata, however the higher uptake were obtained with $S$. heterogama and $A$. muricata. This study showed that several arbuscular mycorrhiza fungi were effective in increasing 
COSTA, N.L. et al. Resposta de Sesbania sesban à inoculação de micorrizas arbusculares. PUBVET, Londrina, V. 6, N. 9, Ed. 196, Art. 1314, 2012.

DM yield, and $\mathrm{N}$ and $\mathrm{P}$ uptake of $\mathrm{S}$. sesban. The most efficient species were $S$. heterogama and $A$. muricata.

Keywords: dry matter, nitrogen, phosphorus, root colonization

\section{Introdução}

O desenvolvimento de sistemas agrícolas mais sustentáveis sob o ponto de vista técnico, econômico, social e ambiental é uma das alternativas para a substituição da agricultura itinerante, caracterizada pelo binômio derruba e queima. A utilização de leguminosas arbustivas, na recuperação de solos degradados ou na melhoria daqueles de baixa fertilidade natural, tem sido uma prática usual nas regiões tropicais, notadamente nas áreas destinadas à produção de alimentos básicos.

Dentre as leguminosas arbustivas avaliadas na Amazônia Ocidental, Sesbania sesban destacou-se entre as mais promissoras, em decorrência de sua boa adaptação a solos de baixa fertilidade, elevado rendimento de biomassa e altos teores de nutrientes (COSTA et al., 1998). Esta leguminosa apresenta crescimento rápido, adapta-se em locais com precipitação entre 500 e $2000 \mathrm{~mm}$ e altitudes até $2000 \mathrm{~m}$. Ademais, é utilizada na alimentação de ruminantes e adubação verde (ASH, 1990; KAITHO et al., 1996; FERREIRA et al., 2002; PAULINO et al., 2008). Gutteridge e Shelton (1991) estudaram a introdução de faixas de $4 \mathrm{~m}$ de largura de $S$. sesban em pastagens com Brachiaria decumbens. Os resultados para B. decumbens $+S$. sesban foram excelentes para os aumentos no ganho de peso vivo, semelhantes aos obtidos com braquiária adubada com $200 \mathrm{~kg}$ de N/ha/ano, entretanto o pastejo causou quebra de muitos ramos devido a sua fragilidade.

Por outro lado, considerando-se que o fósforo é dos fatores mais limitantes ao crescimento das leguminosas (SYLVESTER-BRADLEY et al., 1982; COSTA et al., 2007), a inoculação de micorrizas arbusculares é uma alternativa para aumentar a disponibilidade de fósforo e sua absorção pelas plantas. Os efeitos positivos da micorrização sobre o crescimento de leguminosas dos gêneros Cajanus, Leucaena, Acacia e Desmodium foram relatados em diversos 
COSTA, N.L. et al. Resposta de Sesbania sesban à inoculação de micorrizas arbusculares. PUBVET, Londrina, V. 6, N. 9, Ed. 196, Art. 1314, 2012.

trabalhos. Contudo, as respostas são condicionadas às interrelações entre características do solo, espécies de leguminosas e fungos micorrízicos (COSTA et al., 1991; COSTA; PAULINO, 1997).

Este trabalho teve como objetivo avaliar o efeito da inoculação de micorrizas arbusculares sobre a produção de biomassa e composição química de Sesbania sesban.

\section{Material e Métodos}

O ensaio foi conduzido em casa-de-vegetação, utilizando-se um Latossolo Amarelo, textura argilosa, com as seguintes características químicas: $\mathrm{pH}$ em água $(1: 2,5)=4,8 ; \mathrm{Al}=1,9 \mathrm{cmol} / \mathrm{dm}^{3} ; \mathrm{Ca}+\mathrm{Mg}=1,4 \mathrm{cmol} / \mathrm{dm}^{3} ; \mathrm{P}=$ $2 \mathrm{mg} / \mathrm{kg}$ e $\mathrm{K}=71 \mathrm{mg} / \mathrm{kg}$. O solo foi coletado na camada arável (0 a $20 \mathrm{~cm}$ ), destorroado e passado em peneira com abertura de $6 \mathrm{~mm}$, sendo a seguir esterilizado em autoclave a $110^{\circ} \mathrm{C}$, por uma hora com intervalos de 24 horas, durante três dias, a vapor fluente e pressão de 1,5 atm.

O delineamento experimental foi em blocos casualizados com quatro repetições, sendo os tratamentos constituídos por seis espécies de micorrizas arbusculares (MA): Glomus mossaea, Glomus fasciculatum, Glomus macrocarpum, Glomus etunicatum, Acaulospora muricata e Scutellospora heterogama. Cada unidade experimental constou de um vaso com capacidade para 3,0 kg de solo seco. A inoculação das MA foi realizada adicionando-se 15 $\mathrm{g}$ de inóculo/vaso (solo + esporos + raízes), contendo aproximadamente 400 esporos $/ 50 \mathrm{~g}$ de solo, o qual foi colocado numa camada uniforme cerca de 5 $\mathrm{cm}$ abaixo do nível de plantio. Após o desbaste, deixaram-se duas plantas/vaso.

O controle hídrico foi realizado diariamente através da pesagem dos vasos, mantendo-se o solo em $80 \%$ de sua capacidade de campo. Doze semanas após o desbaste, as plantas foram cortadas rente ao solo, postas para secar em estufa à $65^{\circ} \mathrm{C}$, por 72 horas, sendo a seguir pesadas e moídas em peneira de $2,0 \mathrm{~mm}$. As taxas de colonização radicular foram avaliadas através da observação, ao microscópio, de 20 fragmentos de raízes com $2 \mathrm{~cm}$ 
COSTA, N.L. et al. Resposta de Sesbania sesban à inoculação de micorrizas arbusculares. PUBVET, Londrina, V. 6, N. 9, Ed. 196, Art. 1314, 2012.

de comprimento, clarificadas com $\mathrm{KOH}$ e tingidas por azul de tripano em lactofenol, segundo a técnica de Phillips e Hayman (1970).

\section{Resultados e Discussão}

A análise estatística revelou significância $(P<0,05)$ para o efeito da micorrização sobre os rendimentos de matéria seca (MS). Entre os fungos avaliados, os mais eficientes foram $S$. heterogama e $A$. muricata, os quais proporcionaram incrementos de 155 e 140\%, respectivamente, em relação ao tratamento sem inoculação. Já, G. mossaea foi o fungo menos efetivo, contudo apresentou um acréscimo na produção de MS de 45\%, comparativamente ao tratamento testemunha (Figura 1).

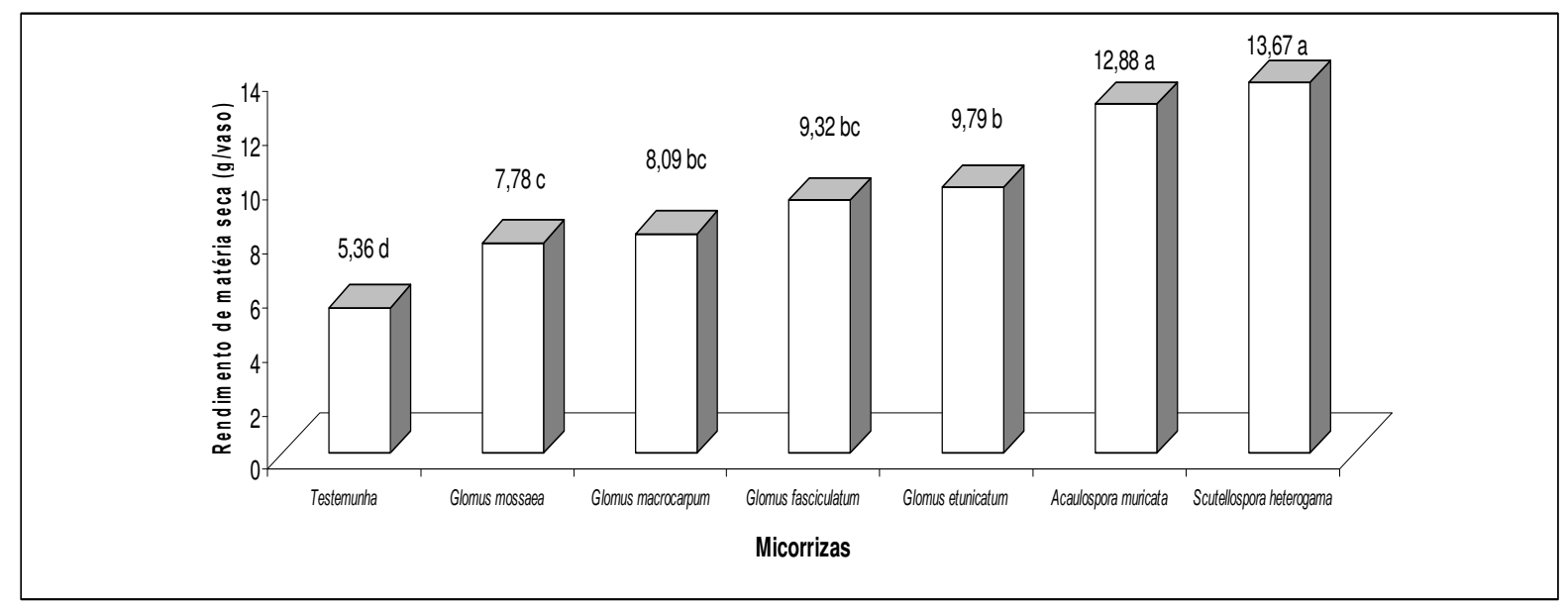

Figura 1. Rendimento de matéria seca (MS) de Sesbania sesban, em função da inoculação de micorrizas arbusculares.

Da mesma forma, Paulino et al. (1992) constataram diferenças significativas na efetividade de quatro espécies de MA no rendimento de forragem de Centrosema brasilianum, sendo os maiores valores obtidos com a inoculação de A. muricata e Gigaspora margarita. Costa et al. (1991) com L. leucocephala, observaram maior efetividade de A. muricata e S. heterogama, comparativamente a G. fasciculatum, G. macrocarpum e G. margarita. Segundo Kruckelmann (1975) as plantas apresentam grande variabilidade na 
COSTA, N.L. et al. Resposta de Sesbania sesban à inoculação de micorrizas arbusculares. PUBVET, Londrina, V. 6, N. 9, Ed. 196, Art. 1314, 2012.

resposta à inoculação de MA, a qual parece ser controlada geneticamente, através de variações fisiológicas dos endófitos e dos mecanismos de infecção, podendo ocorrer especificidade até mesmo ao nível de variedades e/ou cultivares.

As taxas de colonização radicular foram afetadas $(P<0,05)$ pelas diferentes espécies de MA. Os maiores valores foram registrados com a inoculação de G. fasciculatum, S. heterogama e G. mossaea (Figura 2). O mecanismo que regula a relação entre infecção das raízes por MA não é ainda bem conhecido, porém deve estar associado ao nível crítico interno de fósforo da planta hospedeira. Neste trabalho observou-se esta tendência, pois maiores taxas de colonização radicular não refletiram, necessariamente, em maiores teores de fósforo nos tecidos das plantas.

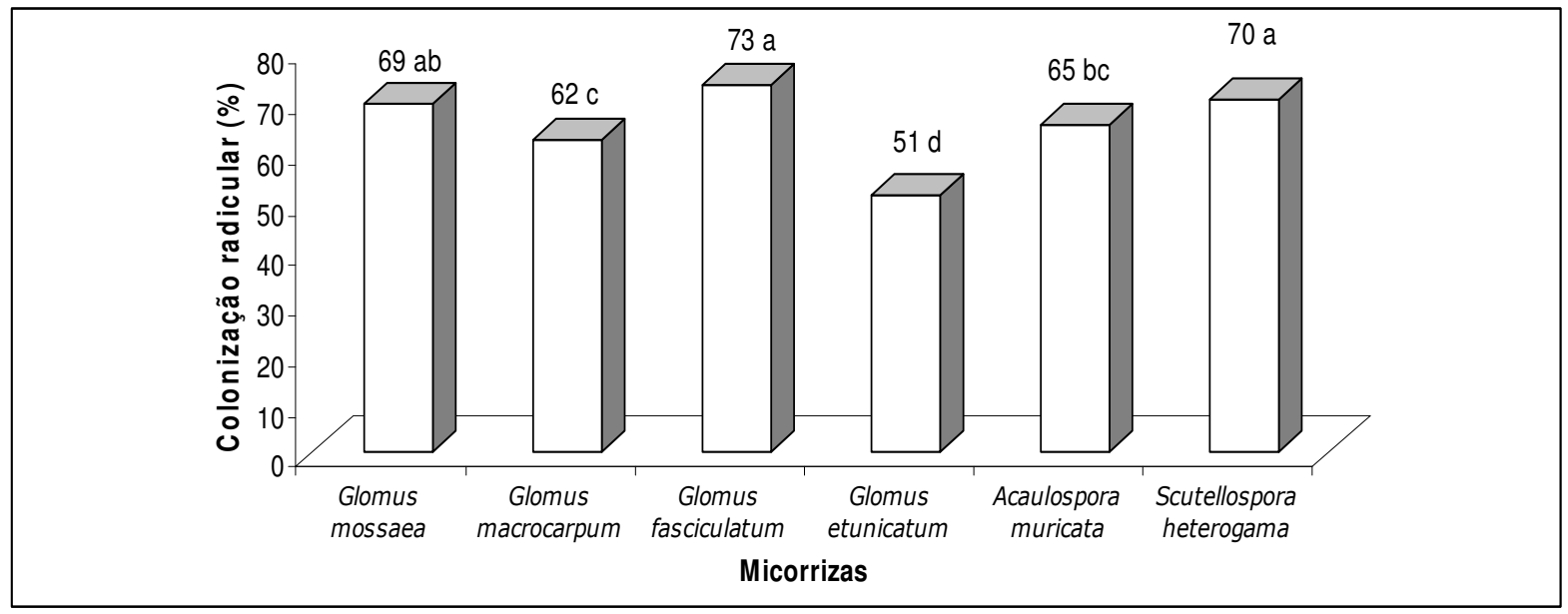

Figura 2. Colonização radicular de Sesbania sesban, em função da inoculação de micorrizas arbusculares.

Miranda et al. (1989) demonstraram que existe um balanço entre o fósforo $(P)$ do solo e do tecido que controla esta relação simbiótica. O efeito do $P$ do solo seria provavelmente mais evidente na fase inicial de colonização radicular, quando o fungo está se desenvolvendo no solo, seja na germinação dos esporos ou no crescimento micelial anterior à penetração na raiz. De acordo com Green et al. (1976), geralmente as espécies dos gêneros 
COSTA, N.L. et al. Resposta de Sesbania sesban à inoculação de micorrizas arbusculares. PUBVET, Londrina, V. 6, N. 9, Ed. 196, Art. 1314, 2012.

Gigaspora, Scutellospora e Acaulospora ocorrem em uma faixa maior de $\mathrm{pH}$, apresentando melhor adaptação e maior efetividade em solos ácidos que as de Glomus.

Os maiores teores de nitrogênio ( $N$ ) foram obtidos com a inoculação de G. mossaea e G. etunicatum, enquanto que plantas micorrizadas por $S$. heterogama e $A$. muricata apresentaram as maiores quantidades absorvidas de $\mathrm{N}$. Para os teores de $\mathrm{P}$, os maiores valores foram verificados com a inoculação de G. mossaea e A. muricata, no entanto a sua maior absorção ocorreu com a inoculação de $S$. heterogama e $A$. muricata (Tabela 1). O estímulo no crescimento da planta atribuído aos fungos micorrízicos estão fortemente correlacionados com o maior acúmulo de nutrientes, especialmente o P. As plantas micorrizadas são favorecidas, pois as hifas do fungo podem beneficiarse do $P$ distante da rizosfera que permanece inacessível às plantas não micorrizadas. Trabalhos conduzidos poro Munns e Mosse (1980) e Costa et al. (1991) reforçam a importância das MA em satisfazer os requerimentos de fósforo pelo Rhizobium durante os processos de nodulação e fixação de N. O incremento na absorção de nitrogênio provocado pela inoculação de MA corroboram com esta idéia, uma vez que na ausência de inoculação as quantidades de nitrogênio acumuladas foram baixas.

Tabela 1. Quantidades absorvidas de nitrogênio e fósforo de Sesbania sesban, em função da inoculação de micorrizas arbusculares.

\begin{tabular}{lccccc}
\hline \multirow{2}{*}{ Tratamentos } & \multicolumn{2}{c}{ Nitrogênio } & & \multicolumn{2}{c}{ Fósforo } \\
\cline { 2 - 3 } \cline { 6 - 7 } & $\mathrm{g} / \mathrm{kg}$ & $\mathrm{mg} / \mathrm{vaso}$ & & $\mathrm{g} / \mathrm{kg}$ & $\mathrm{mg} / \mathrm{vaso}$ \\
\hline Testemunha & $31,18 \mathrm{ab}$ & $16,71 \mathrm{~d}$ & & $1,46 \mathrm{~d}$ & $0,78 \mathrm{~d}$ \\
Glomus macrocarpum & $28,45 \mathrm{bc}$ & $23,01 \mathrm{c}$ & & $1,57 \mathrm{c}$ & $1,27 \mathrm{c}$ \\
Glomus fasciculatum & $27,98 \mathrm{c}$ & $26,08 \mathrm{c}$ & & $1,54 \mathrm{c}$ & $1,43 \mathrm{bc}$ \\
Glomus etunicatum & $31,08 \mathrm{ab}$ & $30,42 \mathrm{~b}$ & & $1,55 \mathrm{c}$ & $1,52 \mathrm{~b}$ \\
Glomus mossaea & $34,19 \mathrm{a}$ & $26,60 \mathrm{c}$ & & $1,75 \mathrm{a}$ & $1,36 \mathrm{bc}$ \\
Acaulospora muricata & $27,06 \mathrm{c}$ & $34,85 \mathrm{a}$ & & $1,70 \mathrm{a}$ & $2,19 \mathrm{a}$ \\
Scutellospora heterogama & $28,13 \mathrm{bc}$ & $38,45 \mathrm{a}$ & & $1,64 \mathrm{~b}$ & $2,24 \mathrm{a}$ \\
\hline - Médias seguidas de mesma letra não diferem entre si (P > 0,05) pelo teste de Tukey
\end{tabular}


COSTA, N.L. et al. Resposta de Sesbania sesban à inoculação de micorrizas arbusculares. PUBVET, Londrina, V. 6, N. 9, Ed. 196, Art. 1314, 2012.

\section{Conclusões}

Os resultados obtidos evidenciam que a inoculação de micorrizas arbusculares incrementa significativamente os rendimentos de matéria seca, teores e quantidades absorvidas de nitrogênio e fósforo da leguminosa, sendo que os fungos mais efetivos, em termos de rendimento de matéria seca, foram S. heterogama e A. muricata.

\section{Referências Bibliográficas}

ASH, A.J. The effect of supplementation with leaves from the leguminous trees Sesbania grandiflora, Albizia chinensis and Gliricidia sepium on the intake and digestibility of Guinea grass hay by goats. Animal Feed Science and Technology, v.28, p.225-232, 1990.

COSTA, N. de L.; PAULINO, V.T.; VEASEY, E.A.; LEÔNIDAS, F. das C. Growth responses of leucaena to vesicular-arbuscular mycorrhizal inoculation. Leucaena Research Reports, v.12, p.12-13, 1991.

COSTA, N. de L.; PAULINO, V.T. Growth response of Acacia angustissima to vesiculararbuscular mycorrhizal inoculation. Forest, Farm and Community Tree Research Reports, v.4, p.51-53, 1997.

COSTA, N. de L.; LEÔNIDAS, F. das C.; TOWNSEND, C.R.; MAGALHÃES, J.A. VIEIRA, A.H. Avaliação de leguminosas arbóreas e arbustivas de múltiplo uso em Rondônia. Porto Velho: Embrapa Rondônia, 11p. 1998. (Embrapa Rondônia. Boletim de Pesquisa, 27).

COSTA, N. L.; PAULinO, V. T.; TOWNSEND, C. R.; MAgALHAES, J. A. Resposta de Arachis pintoi cv. Amarillo à níveis de fósforo. Revista de Biologia e Ciências da Terra, v.6, n.1, p. 59-62, 2006.

FERREIRA, C.A.G.; TAUK-TORNISIELO, S.M.; CHAVES, M.M.F. Comportamento de bracatinga (Mimosa scabrella) e sesbânia (Sesbania sesban) em áreas degradadas pela disposição de resíduos provenientes do processamento da bauxita. Holos Environment, v. 2, n. 2, p. 156 $173,2002$.

GREEN, N.E.; GRAHAM, S.S.; SCHENCK, N.C. The influence of $\mathrm{pH}$ on the germination of vesiculararbuscular mycorrhiza spores. Mycologia, v.68, p.929-934, 1976.

GUTTERIDGE, R.C.; SHELTON, H.M. Evaluation of Sesbania sesban - a new forage shrub species for tropical and subtropical Australia. Final Technical Report, Meat Research Corporation, Canberra. 12p. 1991.

KAITHO, R.J.; UMUNNA, N.N.; NSAHLAI, I. V.; TAMMINGA, S; VABRUCHEM, J.; HANSON, J.; VANDEWOUW, M. Palatability of multipurpose tree species. Effect of species and length of study on intake and relative palatability by sheep. Agroforestry Systems, v.33, n.3, p. 249$261,1996$.

KRUCKELMANN, H.W. Effects of fertilizers, soils, soil tillage and plant species on the frequency of Endogone chlamydospores and mycorrhizal infection in arable soil. In: SANDERS, F.E.; 
MOSSE, B.; TINKER, P.B. (Eds.) Endomycorrhizas. London: Academic Press, 1975. p.511526.

MIRANDA, J.C.C.; HARRIS, P.J.; WILD, A. Effects of soil and plant phosphorus concentrations on vesicular-arbuscular mycorrhiza in sorghum plants. New Phytologist, v.12, p.405-410, 1989.

MUNNS, D.N.; MOSSE, B. Mineral nutrition of legume crops. In: SUMMERFIELD, J.; BUNTING, A.H. (Eds.). Advances in legume sciences. Kew (UK): Royal Botanic Gardens, 1980. p.115125.

PAULINO, V.T.; BRAGA, G.J.; LUCENA, M.A.C.; GERDES, L.; COLOZZA, M T. Sustentabilidade de pastagens consorciadas - ênfase em leguminosas forrageiras. In: ENCONTRO SOBRE LEGUMINOSAS FORRAGEIRAS 2. Anais... Nova Odessa: IZ/APTA/SAA, 2008, v. 1, p. 1-55.

PAULINO, V.T.; COSTA, N. de L.; RODRIGUES, A.N.A.; CHAGAS, F. das. Eficiência de fungos micorrízicos vesículo-arbusculares e da adubação fosfatada em Centrosema brasilianum (L.)Benth. Pasturas Tropicales, v.14, n.3, p.14-17, 1992.

PHILLIPS, J.M.; HAYMAN, D.S. Improved procedure for clearing roots and staining parasitic and vesiculararbuscular mycorrhizal fungi for rapid assessement for infection. Transactions of the British Mycological Society, v.55, p.158-161, 1970.

SYLVESTER-BRADLEY， R.; ASAKAWA, N.; LA TORRACA, S.M.; MAGALHÃES, F.M.M.; OLIVEIRA, L.A.; PEREIRA, R.M. Levantamento quantitativo de microorganismos solubilizadores de fosfato na rizosfera de gramíneas e leguminosas forrageiras na Amazônia. Acta Amazonica, v.12, n.1, p.15-22, 1982. 